\title{
TRABALHO E SAÚDE: EXIGÊNCIAS DA ATIVIDADE DOCENTE NO ENSINO SUPERIOR
}

\author{
WORK AND HEALTH: REQUIREMENTS FOR \\ TEACHING IN HIGHER EDUCATION
}

Cryslaine Vanessa Coutinho dos Reis (Orcid: 0000-0001-9536-068X) ${ }^{1}$ Arlete Ana Motter (Orcid: 0000-0002-2585-207X)²

\section{Contato}

Arlete Ana Motter

E-mail: arlete.motter@uol.com.br

${ }^{1}$ Programa de Residência Multiprofissional em Saúde da Família, Secretaria Municipal da Saúde de Curitiba, Fundação Estatal de Atenção à Saúde e Faculdades Pequeno Príncipe, Paraná, Brasil.

${ }^{2}$ Curso de Fisioterapia, Universidade Federal do Paraná, Paraná, Brasil.

\section{RESUMO}

Introdução: o trabalho na docência, considerado uma das mais antigas profissões, exige a execução de diversas atividades que não envolvem apenas ensino, pesquisa e extensão, mas também abrangem os aspectos físicos, psíquicos e cognitivos influenciando no processo saúde-doença. Objetivo: analisar as exigências físicas, mentais e os riscos psicossociais presentes na atividade docente em uma universidade pública no Brasil. Métodos: a pesquisa foi aprovada pelo Comitê de Ética em Pesquisa do Setor de Ciências da Saúde da Universidade Federal do Paraná sob número: 1.175.594. Para a seleção amostral, foi realizado um cálculo com base na estimativa da população proporcional. Fizeram parte do estudo 66 participantes. Na primeira etapa, foi utilizado o instrumento Questionário Saúde e Trabalho em Atividades de Serviço (QSATS). Na segunda, foram realizadas entrevistas semiestruturadas com questões relacionados com os aspectos psicossociais do trabalho, as quais foram gravadas e transcritas na íntegra para posterior análise. Resultados: a amostra foi constituída por 66 docentes, sendo 33,33\% da população masculina; e 66,67\%, feminina. A média de idade foi de 42,5 $\pm 9,20$ anos. Em relação ao tempo de atuação na instituição, a média foi de $9,54 \pm 8,91$ anos. Os resultados demonstraram que os docentes estão constantemente vulneráveis aos constrangimentos e ao adoecimento no que se refere à sobrecarga, à infraestrutura, ao relacionamento interpessoal e à desvalorização profissional, interferindo diretamente no processo saúde-doença. Conclusão: há, sobretudo, uma realização pessoal e satisfação pelo amor ao exercício docente, tornando esta uma relação dialética entre o prazer e o sofrimento.

Palavras-chave: Trabalho; Professores; Universidade; Saúde.

\begin{abstract}
Introduction: teaching work, considered one of the oldest professions, requires the execution of several activities that not only involve teaching, research and extension, but also encompass the physical, psychological, and cognitive aspects influenced by the health-disease process. Objective: to analyze the physical, mental, and psychosocial risks requirements for teaching activities at a public university in Brazil. Methods: a research was approved by the Research Ethics Committee of the Health Sciences Sector of the Federal University of Paraná under the number: $1,175,594$. For a sample selection, a calculation was performed based on the proportional population estimate. 66 participants took part in the study. In the first stage, the questionnaire Health and Work in Activities of the QSATS Service was used. In the second, semi-structured interviews were conducted with questions related to the psychosocial aspects of work, which were recorded and transcribed in full for later analysis. Results: the sample consisted of 66 professors, being $33.33 \%$ of them male and $66.67 \%$ female. The mean age was $42.5 \pm 9.20$ years Regarding the length of experience at the institution, the average was $9.54 \pm 8.91$ years. The results demonstrated that professors are vulnerable to constraints and changes with regard to overload, infrastructure, interpersonal relationships, and professional devaluation, directly interfering in the health-disease process. Conclusion: Above all, there is personal fulfillment and satisfaction regarding the love for teaching, promoting a dialectical relationship between pleasure and suffering.
\end{abstract}

Keywords: Work; Teachers; Universities; Health 


\section{8}

\section{INTRODUÇÃO}

A palavra "trabalho" possui diferentes contextos que podem refletir nas condições de trabalho, nos resultados e na atividade a ser realizada ${ }^{1}$. A docência, considerada uma das mais antigas profissões, exige a execução de diversas tarefas e atividades que abrangem aspectos físicos, psíquicos e cognitivos e que influenciam no processo saúde-doença².

O docente universitário, em específico, possui um papel diferente no processo ensino-aprendizagem, pois seu trabalho vai além do ensino em sala de aula, iniciando enquanto estudante até sua qualificação profissional, que, muitas das vezes, é contínua e estende-se ao longo da carreira. Para Canan et al., o professor universitário não depende apenas dos aspectos técnicos ou administrativos para exercer sua função, mas também do autoconhecimento e do desenvolvimento biopsicossocial como indivíduoº

Nesse contexto, o desenvolvimento desses profissionais, para Melo et al., excede o processo como um desenvolvimento global humano ou de autoformação ${ }^{4}$, não podendo ser considerado apenas como um produto por sua complexidade, resultando, assim, em um processo ensino -aprendizagem por meio de trocas de conhecimentos, saberes, práticas, experiências e até mesmo crenças pessoais, fatores que interferem no processo saúde-doença dos docentes ${ }^{3}$.

A rotina intensa de trabalho associada às exigências por produção, ao aperfeiçoamento técnico científico, à for- mação continuada, às publicações científicas, à geração de resultados, além da responsabilidade de formar indivíduos como futuros profissionais preparados ao mercado de trabalho, tornam os docentes expostos ao adoecimento. Além disso, o exercício da função exige competências técnicas, mas, sobretudo, o enfrentamento de condições precárias e de políticas públicas de uma universidade federal ${ }^{4}$.

Sabe-se que a condição de saúde e o trabalho estão diretamente relacionados com a qualidade de vida e com o desempenho profissional. Nos últimos anos, houve um crescimento de problemas afetando a saúde dos professores, os quais estão associados às condições de trabalho $\mathrm{e}$ afetam tanto a saúde física quanto a saúde mental dessa classe profissional ${ }^{3}$.

Os professores universitários prejudicam constantemente sua qualidade de vida por diversos fatores, como: condições ambientais, físicas e organizacionais. Essas características os deixam constantemente sujeitos às tensões provenientes da responsabilidade do processo ensino-aprendizagem. Diante disso, além das exigências ergonômicas que geram sintomas osteomusculares, destacam-se outros distúrbios que afetam a saúde ocupacional dos professores universitários, como o estresse, a depressão e a Síndrome de Burnout ${ }^{3-5}$.

Ressalta-se que as responsabilidades dos docentes vão além das atividades de ensino, pesquisa e extensão, visto que envolvem também atividades administrativas. Isso pode desencadear ao trabalhador uma insatisfação profissional, 
expondo-os aos altos níveis de estresse $\mathrm{e}^{6-7}$. Carlotto et al. ${ }^{8}$ citam outros fatores que prejudicam a atividade laboral dos professores universitários: a insatisfação salarial e a falta de reconhecimento?.

Além disso, nas últimas décadas, houve uma transformação da relação entre sociedade e universidade, em que os docentes sofrem os impactos de forma negativa, visto que novos modelos pedagógicos foram implementados, além das mudanças nas políticas e no processo de gestão das universidades federais, que também influenciam na dinâmica e no cotidiano de trabalho ${ }^{10-11}$.

Segundo Arruda et al., há uma desvalorização social em relação à importância da atividade exercida pelos professores, associada ao aumento das exigências da profissão, pois, além da exigência por uma formação contínua e permanente, é necessário que o professor universitário compreenda a prática da docência para que o processo de ensino seja eficaz. Sendo assim, existe uma preocupação e uma autocobrança ante a responsabilidade de capacitar com qualidade estudantes a futuros profissionais de sucesso ${ }^{10}$.

Nesse contexto, o impacto dessas condições físicas, psíquicas, cognitivas e sociais na execução do trabalho estão associadas ao desgaste físico e emocional desses profissionais, tornando-se um problema de saúde pública. Campos et al. citam que os transtornos mentais e com- portamentais são as principais queixas de saúde de docentes universitários, sendo a terceira principal causa de afastamento de trabalho por incapacidade entre esses profissionais. Entretanto, o impacto não é somente na saúde psíquica dos docentes, mas, sim, econômico e social ${ }^{11}$.

O trabalho educacional para esses profissionais, portanto, é considerado uma constante relação entre o prazer e o sofrimento, uma vez que eles também se sentem satisfeitos pelo amor ao exercício docente ${ }^{11-12}$. Cortez et al. enfatizam a importância de outros estudos para compreender o processo saúde-doença na docência com o intuito de contribuir para a melhora das condições de trabalho dessa categoria profissional ${ }^{13}$.

Diante disso, o objetivo do estudo é analisar as exigências físicas, mentais e os riscos psicossociais presentes na atividade docente em uma universidade pública no Brasil.

\section{MÉTODOS}

Trata-se de um estudo transversal, exploratório e descritivo. Desenvolveu-se entre agosto de 2016 e julho de 2018, em Curitiba/Paraná. O projeto foi aprovado pelo Comitê de Ética em Pesquisa do Setor de Ciências da Saúde em 7 de agosto de 2015, sob o número: 1.175.594. 
A pesquisa foi realizada na Universidade Federal do Paraná (UFPR). Os critérios de inclusão foram: ser professor efetivo do setor em que a pesquisa se desenvolveu; de qualquer idade; de ambos os gêneros; que concordassem em participar e que assinassem o Termo de Consentimento Livre e Esclarecido (TCLE). Os critérios de exclusão foram: não pertencer ao setor; ser professor substituto; estar de licença; não concordar em participar do estudo ou não devolver o questionário respondido.
Após um cálculo amostral com base na estimativa da proporção populacional, o resultado obtido foi de uma amostra de 156 participantes, sendo o setor formado ao todo por cerca de 250 docentes. Entretanto, apenas 62 aceitaram participar da primeira etapa da pesquisa; e da segunda, 4 docentes (Figura 1).

Figura 1. Seleção da amostra

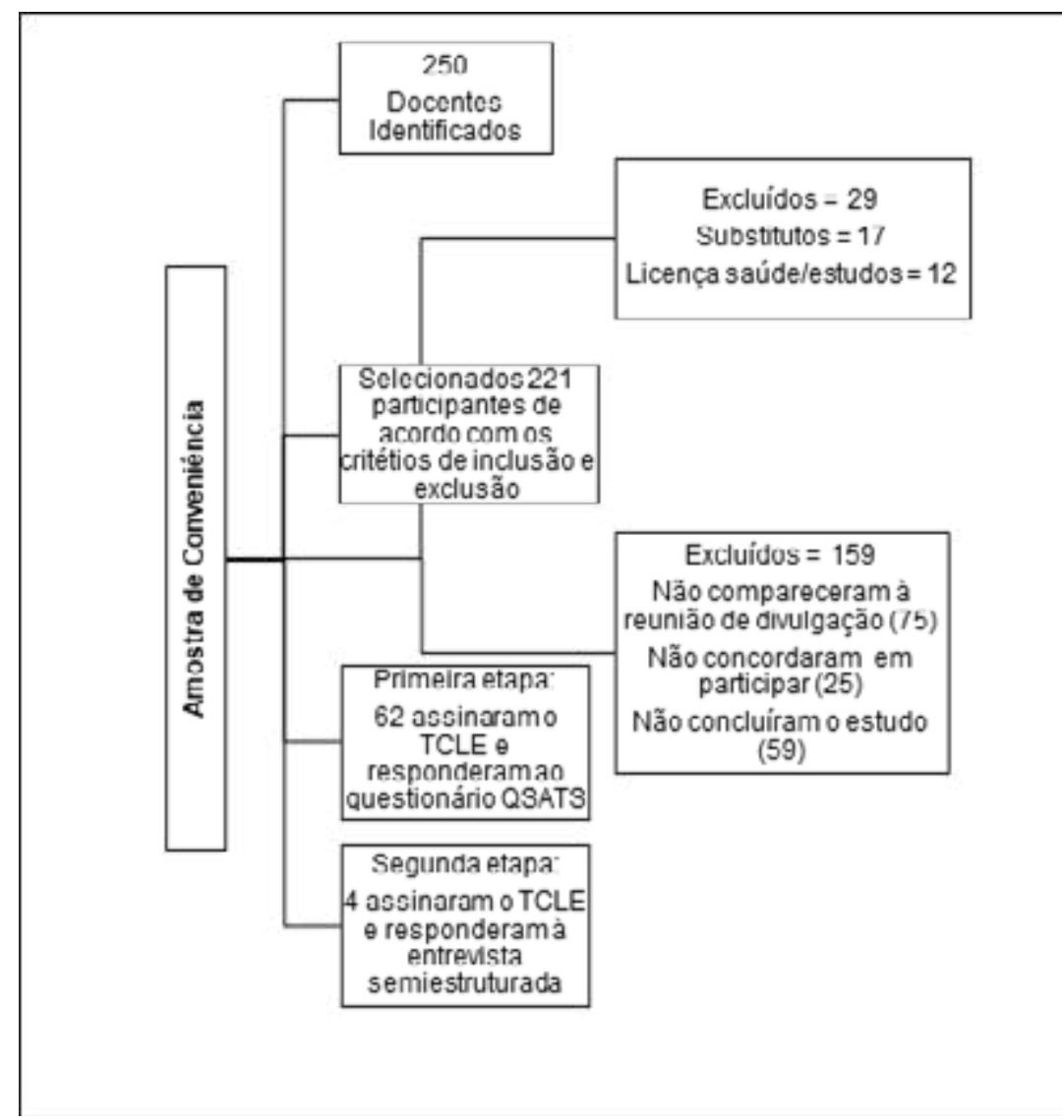




\section{Etapas da seleção amostral}

Para a coleta de dados da primeira etapa, foi aplicado o instrumento Questionário Saúde e Trabalho em Atividades de Serviço (QSATS) (2015), questionário autoaplicável e adaptado pela equipe pesquisadora, sendo composto pelas seguintes questões: Identificação geral; Condições e características do trabalho; Aspectos ambientais; Aspectos físicos; Ritmo e intensidade de trabalho; Vida familiar e lazer e Problemas de saúde e, por último, questões abertas, como: As minhas condições de trabalho são satisfatórias?; Minha remuneração é compatível com minhas atribuições e responsabilidades?; Há exigências por produção científica?; Aspectos que considero positivos em meu trabalho; Aspectos mais problemáticos (crítico ou negativos) de meu trabalho.

Além disso, na segunda etapa, foram realizadas entrevistas individuais, as quais foram gravadas e transcritas na íntegra para análise, sendo constituídas por cinco questões: 1) De acordo com suas atribuições e responsabilidades, você se sente valorizada profissionalmente? 2) Quais são as principais dificuldades que você encontra no seu trabalho? 3) Existe uma pressão por produção científica? 4) Além de suas funções habituais, você exerce funções administrativas? Se sim, isso afeta positivamente ou negativamente seu trabalho? 5) Como você julga suas relações com os colegas de trabalho? Se sente parte de uma equipe?
Com o intuito de preservar a identidade dos participantes, na apresentação dos resultados, adotou-se um código, no qual " $\mathrm{D}$ ” corresponde a Docente seguido de um número entre 1 e 66 (amostra).

\section{RESULTADOS E DISCUSSÃO}

Os resultados apresentados a seguir contemplam variáveis do perfil sociodemográfico da população estudada, bem como questões psicossociais relacionados com o trabalho docente, obtidas por meio do instrumento QSATS (2015). Os dados obtidos por meio desse instrumento concentraram-se nas respostas abertas, visto que os demais foram analisados em outro estudo realizado por Molina et $\mathrm{al}^{14}$.

Participaram da pesquisa 66 professores do Setor de Ciências Biológicas da UFPR, sendo $33,33 \%$ da população masculina, e $66,67 \%$, feminina. A faixa etária foi entre 30 anos e 68 anos, com a média de idade de 42,5 $\pm 9,20$ anos. Em relação ao tempo de atuação na institui-

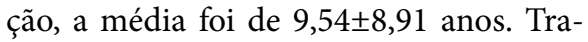
tando-se da jornada de trabalho, nota-se o predomínio de docentes com o regime de dedicação exclusiva à universidade, sendo 95,16\% da população estudada.

Quanto à carga horária semanal em sala de aula dos participantes da primeira etapa, a média encontrada foi de 9,8 horas-aula. Contudo, mesmo com cargas horárias extensas, observou-se que 
93,54\% dos entrevistados relataram realizar atividades laborais em casa, e 96,77\% afirmaram que se preocupam com o trabalho fora da universidade.

\section{CONDIÇÕES DE TRABALHO: INFRAESTRUTURA}

Para realizar o trabalho docente, é necessário um espaço físico adequado, pois, além da carga horária semanal em sala de aula, os professores precisam executar as demais atividades laborais. Assim, a infraestrutura é uma das condições de trabalho que, em circunstâncias precárias, pode provocar uma situação estressante, a ponto de os trabalhadores se sentirem insatisfeitos e considerarem tal situação constrangedora ${ }^{15}$, conforme demonstram os relatos:

\footnotetext{
"Sala coletiva (gabinete) em espaço pequeno. Sala com infiltrações e muito bolor. Mobiliário inadequado. Laboratório com condições precárias, a universidade não disponibiliza EPIs. Laboratórios didáticos sem ar condicionado, gás encanado, ar condicionado, falta de materiais de um modo geral.” (D35)
}

"Estrutura física ruim, possibilidade de obter materiais de pesquisa e para aulas baixa, falta de equipamentos (computador etc.)...” (D47)

"Trabalho em um prédio cujas condições físicas beiram a insalubridade." (D55)
Além disso, o relato de uma docente enfatiza a questão burocrática no processo para adquirir materiais e recursos para realizar o exercício laboral, principalmente por depender de órgãos específicos para a liberação de recursos financeiros.

\begin{abstract}
"A logística do processo ela é complicada. Eu tô esperando um armário aqui faz uns dois anos, essa semana eu consegui um armário que vai vir de outro setor, tem um monte de coisa que a gente traz de casa mesmo. Então, boa parte das coisas que a gente tem aqui, na verdade são coisas pessoais que você traz para o teu ambiente de trabalho. Isso, pra uma instituição pública deveria ser provido! Em relação ao que a gente faz propriamente dito eu preciso de outros órgãos, pra que esses outros órgãos disponibilizem recurso pra isso." (D64)
\end{abstract}

Um estudo realizado por Motter et al. com docentes de uma universidade pública no sul do Brasil demonstra que essas situações são constantes e fazem com que os profissionais optem por utilizar seus recursos tecnológicos pessoais e até mesmo um espaço físico próprio ${ }^{12}$. Para Gomes et al., as características do local de trabalho, como a tecnologia, os equipamentos e a estrutura física, estão diretamente relacionadas com a qualidade de vida do trabalhador, visto que exercem grande influência na saúde física, cognitiva e psíquica desse profissional ${ }^{4}$. 


\section{ASPECTOS ORGANIZACIO- NAIS E SUAS REPERCUSSÕES NO PROCESSO SAÚDE-DOEN- ÇA}

A carga de trabalho refere-se ao conjunto de esforços desenvolvidos para atender às exigências das tarefas (físicos, cognitivos e psíquicos). A carga quantitativa diz respeito ao volume de trabalho mental exigido dentro de determinado tempo. A carga qualitativa refere-se ao nível de complexidade do trabalho e à possibilidade de aplicação, ao mesmo tempo, dos interesses significativos, experiência, capacidades e potenciais do trabalhador $^{16-17}$.

A sobrecarga no trabalho é uma das principais queixas dos professores universitários, tornando-se fonte de desgaste e sofrimento. Isso ocorre devido à grande demanda exigida, em contrapartida à falta de infraestrutura e equipamentos adequados para o exercício laboral ${ }^{18}$. Além de estar associada à falta de infraestrutura e recursos, pode-se relacionar a sobrecarga com a pressão por produção científica; essas limitações levam os professores a relatarem insatisfações devido ao excesso de funções e atividades a serem executadas, $o$ que se observa por meio dos relatos:

"Muita demanda administrativa, pressão para ser produtiva diante da intensa competição por recursos, trabalho interminável... Quem não produz rapidamente recebe muito pouco auxílio financeiro." (D2)
“Temos que atuar em várias linhas diferentes administração, pesquisa, graduação. As condições não são as ideais.” (D31)

"Cobrança de publicações, pesquisas e orientações sem contrapartida financeira; acúmulo de funções e cargos (didáticos, administrativos, laboratoriais); pouco apoio técnico-administrativo." (D26)

A pressão por produção científica já se inicia no processo seletivo dos concursos públicos como obrigatoriedade para os cargos de docentes. Além disso, prioriza-se a quantidade de periódicos publicados, o que muitas vezes não se reverte em qualidade. Do mesmo modo, para produzir com qualidade, é necessário recurso financeiro, dificultando o processo das exigências em relação à produção científica.

Nessas circunstâncias, os relatos dos docentes coincidem com o fato de sentirem-se desvalorizados e sobrecarregados, pois, além disso, há a responsabilidade que envolve o trabalho no coletivo, visto que a publicação de periódicos também implica a avaliação do curso e o reconhecimento pelo Ministério da Educação (MEC); e, no caso da pós-graduação stricto sensu, pela Coordenação de Aperfeiçoamento de Pessoal de Nível Superior (Capes). 
"Porque tudo vira número né?! Vem a Capes avaliar então ele não quer saber da qualidade da pessoa, quer tudo transformado em números, então eu acho esse sistema muito ruim... Muita pressão e pouco reconhecimento. Tem gente que tem um tremendo currículo, tenta mandar o projeto e o projeto é rejeitado, tenta conseguir uma verba, não consegue, então é complicado." (D63)

"Não adianta só você produzir, tem que produzir com qualidade, pra produzir com qualidade você precisa de dinheiro." (D64)

Motter et al. descrevem que a sobrecarga no trabalho pode acarretar consequências na saúde física e psíquica dos docentes, visto que, mesmo na tentativa de executar todas as atividades durante a jornada de trabalho, há uma invasão da vida privada ${ }^{12}$. Assim, os profissionais também se sentem sobrecarregados devido ao excesso da carga horária - segundo o relato de uma docente, a classe é desvalorizada para a realidade do exercício profissional. Outros relatos enfatizam o descontentamento nesse sentido:

"Jornada intensa que exige, para manter produtividade, mais de 40h/semanais." (D36)

"Excesso de trabalho levado para casa." (D59)

"O trabalho não termina na sala de aula." (D60)
De acordo com Lago et al., ainda existe a exigência de que os profissionais da docência sejam cada vez mais qualificados para que consigam se inserir no mercado de trabalho, todavia, muitos relatam que as condições de trabalho não favorecem a dedicação e a formação acadêmica deles ${ }^{19}$. Para os autores, essa exigência por altos níveis de formação também gera uma sobrecarga, repercutindo de forma negativa na saúde desses profissionais.

\section{RELACIONAMENTO INTER- PESSOAL E COMPETITIVIDA- DE}

O relacionamento interpessoal no trabalho é designado, de acordo com Santana et al., por um convívio no coletivo, o qual estabelece relações de contato entre pessoas em diferentes contextos. Nessa perspectiva, o ambiente acadêmico envolve relações interpessoais entre colegas de equipe e alunos, o que pode refletir de forma positiva ou negativa na prática docente $^{20}$.

Os aspectos positivos envolvem o prazer pelo processo ensino-aprendizagem. Muitos professores alegam que ensinar e compartilhar conhecimentos com os alunos é gratificante, outros relatam que essa experiência proporciona aprendizado e bem-estar, conforme demonstram alguns relatos: 
"Gosto de ministrar aulas e da relação com os alunos, a relação com a academia é extremamente gratificante pessoalmente...Sempre aprendemos nos inter-relacionando com outros, acredito essa ser a parte mais gratificante, aprender ensinando." (D28)

No entanto, nem sempre a relação professor-aluno estabelece esse sentimento. Nesse sentido, Soares et al. destacam que os conflitos ocorrem devido à insatisfação dos alunos com os conteúdos e às dificuldades na aprendizagem, tornando essa uma relação delicada e vulnerável a tensões e aos conflitos ${ }^{21}$. Em contrapartida, o relato de um professor, ao ser questionado sobre os aspectos negativos da profissão, enfatiza que o problema pode estar no desinteresse dos alunos em aprender (D62).

As relações interpessoais (com colegas e/ou estudantes) podem prejudicar a saúde dos docentes e afetar o desempenho profissional. No trabalho coletivo, os entrevistados ressaltam que o relacionamento com os colegas pode ser classificado como um ponto negativo devido à constante competitividade. Para Valente et al., um ambiente competitivo é considerado tenso e apreensivo, transformando-o em um "terreno fértil para o terror psicológico"22.

Além da competitividade, os professores alegam que o individualismo, a falta de colaboração e de compro- metimento de alguns colegas prejudicam o desempenho, tornando o ambiente de trabalho desagradável. Desse modo, um dos relatos cita a necessidade de apoio médico/psicológico para o enfrentamento das dificuldades associadas às relações interpessoais (D16). Outros, enfatizam o problema exposto:

"Ambiente negativo. Pessoas "tóxicas”. (D34)

"Em alguns casos, os comportamentos pouco comprometido com o trabalho de alguns colegas." (D52)

Contudo, qualquer ambiente de trabalho está vulnerável a conflitos interpessoais, pois o exercício laboral depende de um coletivo, sendo assim, sabe-se que cada indivíduo possui suas particularidades. Partindo desse princípio, alguns entrevistados citam que lidar com as adversidades tem seus aspectos positivos:

"É lógico que todo grupamento de pessoas, você tem aquelas pessoas com quem você se entende melhor, aquelas que você não se entende tão bem né?! Então essa variabilidade toda tem em qualquer lugar! Mas, assim, do meu ponto de vista, pessoal e como profissional, eu não tenho um ambiente hostil, acho que o problema é a questão de recurso etc." (D63) 
"Minha relação é tranquila, eu não tenho nenhum problema com nenhum colega específico. Lógico que, às vezes, a gente entra em debates né, que você vai contra a opinião, e isso é normal das relações humanas né, entre pessoas, porque nem todo mundo pensa igual. A falta de recurso também une um pouco as pessoas nesse sentido." (D64)

Logo, o trabalho educacional não envolve apenas a produtividade, mas também o convício com pessoas que possuem opiniões diferentes. Para o bom funcionamento em coletivo, é preciso lidar com as adversidades e particularidades de cada um. Duarte et al. designam a capacidade de diálogo e escuta como uma interação (seja entre colegas de trabalho ou alunos), que favorece a prática docente pela convivência e troca de experiências ${ }^{23}$.

\section{DESVALORIZAÇÃO PROFIS-} SIONAL E INSATISFAÇÃO SALARIAL

A docência exige diversas responsabilidades já expostas, que vão além até mesmo do processo de formação como docente. $\mathrm{Na}$ tentativa de cumprir toda a demanda exigida com produtividade e eficácia, as atividades laborais ultrapassam a vida profissional. Isso desperta nos professores universitários um sentimento de frustação física e emocional, fazendo com que se sintam desvalorizados profissionalmente $^{24}$.
A falta de valorização associada à insatisfação salarial potencializam a desilusão em relação ao exercício profissional, tornando-se um sofrimento. Nesse sentido, ao serem questionados sobre remuneração salarial e compatibilidade com as atribuições e responsabilidades exigidas no trabalho, os professores relataram que se sentem insatisfeitos e desvalorizados:

\section{"Em comparação com outras áreas é pouco valorizada." (D2) \\ "Não se considerarmos a carga horária, dedicação e tempo de preparo até o início da carreira." (D8)}

"Acredito que pela formação que tenho e anos de estudo o meu salário deveria ser melhor." (D20)

"Comparável com minha formação e dedicação deveria receber mais." (D33)

Apesar de alguns docentes se considerarem reconhecidos profissionalmente devido à afinidade por trabalhar na área educacional, isso não é suficiente para que eles se sintam satisfeitos por completo, pois, segundo Araújo et al., a satisfação envolve tanto os aspectos emocionais quanto as condições do ambiente de trabalho ${ }^{25}$. Portanto, condições inadequadas podem repercutir negativamente no desenvolvimento profissional. 


\section{ASPECTOS POSITIVOS: SATIS- FAÇÃO E AMOR AO EXERCÍ- CIO DOCENTE}

Como toda profissão, o exercício docente possui aspectos negativos e positivos. A vocação pela docência, a dedicação, a paciência e o amor ao exercício são considerados aspectos positivos por essa classe profissional. $\mathrm{O}$ fato de formar futuros profissionais para o mercado de trabalho gera satisfação e realização pessoal aos docentes ${ }^{26}$. Muitos dos entrevistados, ao serem questionados sobre os aspectos positivos do trabalho, relataram o fato de gostar da sua profissão. Aprender e compartilhar com os alunos acadêmicos é considerado gratificante para esses profissionais, mesmo em meio aos desafios diários aos quais são submetidos:

"Faço o que mais gosto, sou realizada, mesmo com as dificuldades "técnicas." (D7)

"Gerar conhecimentos, formar pessoas, ter oportunidade de aprender constantemente, trabalhar em ambiente intelectualmente estimulante, fazer o que gosto." (D8)

"Realização pessoal. Contribuições para a formação profissional dos estudantes; contribuições para a ciência." (D59)
A liberdade de atuação no ensino, pesquisa e extensão, assim como a flexibilidade na escolha dos métodos a serem abordados em sala de aula com os alunos, é outro aspecto considerado positivo que leva à realização pessoal na profissão. Segundo Junges et al., o saber ensinar para os professores confere a identidade profissional, visto que são essenciais didática e métodos eficazes para que os conteúdos sejam compreendidos pelos alunos ${ }^{27}$. Assim, quando a aprendizagem é evidente, o docente sente um sentimento de "dever cumprido", o qual gera a realização pessoal e profissional:

"Prazer em fazer algo que gosto, aprendizado contínuo, conhecer pessoas (alunos) novos todos os anos, prazer por ensinar!" (D2)

"Ver o entusiasmo de alguns alunos pela área em que atuo. Ver o sucesso dos alunos orientados por mim e receber o agradecimento deles." (D17)

"Produção de conhecimento e inovação; função social; ganho social e econômico; é o que gosto; aprender e compartilhar." (D53)

"Autonomia para definir cronograma de trabalho. O aprendizado constante. Contato com alunos (poucos) interessados." (D55)

"Satisfação pessoal. Devido à relação com os colegas de trabatho e alunos. Muito gratificante." (D61) 
Essas características levam o profissional docente a uma estreita relação com o prazer, visto que, apesar de todas as dificuldades expostas, muitos não se deixam desanimar pelo amor ao trabalho. De acordo com Ferreira et al., o sentimento de gratidão e satisfação com seu trabalho pode influenciar positivamente no desempenho do trabalhador, mas principalmente interferir nas dimensões do processo saúde-doença ${ }^{28}$.

Motter et al., em um estudo realizado com controladores de tráfego aéreo, caracterizam o prazer como um significado de trabalho, no qual envolve a satisfação em realizar atividades laborais criando uma identidade profissional. Além disso, a atividade laboral está correlacionada com o estado mental dos indivíduos, interferindo não somente na vida profissional, mas, sobretudo, na vida pessoal dos trabalhadores, nesse caso, dos docentes ${ }^{29}$.

Segundo Barbosa et al., ser professor significa enfrentar condições que facilitam ou impedem o aperfeiçoamento na vida profissional, o que envolve questões organizacionais e de legislação trabalhista, principalmente porque diz respeito ao processo de desenvolvimento educacional de um país ${ }^{30}$.

\section{CONCLUSÃO}

O contexto do trabalho docente é complexo e exige dos professores universitários estratégias para o enfrentamento de diversos agentes estressores que influenciam no processo saúde-doença. Apesar dos constrangimentos e da vulnerabilidade ao adoecimento, há uma realização pessoal e satisfação pelo amor ao exercício docente, transformando-a em uma relação entre o prazer e o sofrimento.

Para atender à demanda exigida do ensino, é necessário repensar as condições de trabalho ofertadas a essa categoria profissional. Sugere-se um planejamento organizacional das universidades públicas para diminuir os danos sofridos pelos professores universitários e, consequentemente, pela educação, visto que a realidade deste estudo pode se estender a outras universidades públicas nacionais.

\section{REFERÊNCIAS}

1. Guérin F, Laville A, Daniellou F, Duraffourgm J, Kerguelen A. Compreender o trabalho para transformá-lo - a prática da ergonomia. 1. ed. São Paulo: Editora Edgard Blucher; 2001.

2. Guerreiro NP, Nunes EDFPD, González AD, Mesas AE. Perfil sociodemográfico, condições e cargas de trabalho de professores da rede estadual de ensino de um município da região sul do Brasil. Trab edu saúde. 2016; 14:197-217.

3. Canan SR, Santos CFS. Educação superior em tempos de crise: há espaço nas políticas de educação para a discussão do professor universitário? EccoS Rev Cient. 2019; (48):339-361.

4. Melo GF, Naves MLDP. Desenvolvimento profissional de professores universitários: reflexões a partir de experiências formativas. LES: Ling Educ Socie. 2019; (31):126-148. 
5. Araújo LMN, Santos VEP, Martins CCF, Dantas MSP, Santos NP, Alves KY. A. Estresse no cotidiano universitário: estratégias de enfrentamento de docentes da saúde. J res: fundam care online. 2016; 8(4):4956-4964.

6. Ugwoke SC, Eseadi C, Igbokwe CC, Chiaha GTU, Nwaubani OO, Orji $\mathrm{CT}$, et al. Effects of a rational-emotive health education intervention on stress management and irrational beliefs among technical college teachers in Southeast Nigeria. Medicine (Baltimore). 2017; Aug; 96(31):e7658.

7. Leite AF, Nogueira JAD. Fatores condicionantes de saúde relacionados ao trabalho de professores universitários da área da saúde: uma revisão integrativa. Rev. bras. saúde ocup. 2017; 42:e6.

8. Carlotto MS, Câmara SG. Riscos psicossociais associados à síndrome de burnout em professores universitários. Av Psicol Latinoam. 2017; 35(3):447-457.

9. Leite JL. Publish or perish: the puzzle of academic productivism. Rev. Katálysis. 2017; 20(2):207-215.

10. Arruda RP, Catrib AMF, Brasil CCP, Batista MH, Sampaio PP. The Pain of teaching: considerations regarding educational endeavors. US-China Education Review A. 2015; 5(4):267276.

11. Campos T, Véras RM, Araújo TM. Trabalho docente em universidades públicas brasileiras e adoecimento mental. Rev Docência Ens Sup. 2020; (10):1-19.
12. Motter AA, Grigorio JM, Antonio NKS. M. Atividade docente em uma universidade pública brasileira: prazer ou sofrimento no trabalho? International Journal on Working Conditions. 2015; 00:22-42.

13. Cortez PA, Souza MVRD, Amaral LO, Silva LCAD. Teachers health in the workplace: evidence from recent literature. Cad saúde colet. 2017; 25(1)113-122.

14. Molina GC, Araujo GC, Klostermann GS, Motter AA. Exigências musculoesqueléticas do trabalho docente em uma universidade pública. Cad Educ Saúde Fisioter. 2017; 4(7):7-14.

15. Silveira RCP, Ribeiro IKS, Teixeira LN, Teixeira GS, Melo JMA, Dias SF. Wellness and health of teachers in a public educational institution. J Nurs UFPE on line-ISSN: 1981-8963 2017; 11(Suppl. 3):1481-1488.

16. Neves MY, Seligmann-Silva E, Athayde M. Saúde mental e trabalho: um campo de estudo em construção. In: Araújo A, Neves MY, Athayde M, Alberto MF, organizadores. Cenários do trabalho: subjetividade, movimento e enigma. Rio de Janeiro: DP\&A; 2004. p. 19-49.

17. Wisner A. Por dentro do trabalho. Ergonomia: método \& técnica. São Paulo: FTD/Oboré; 1987.

18. Carlotto MS, Dias SRS, Batista JBV, Diehl L. O papel mediador da autoeficácia na relação entre a sobrecarga de trabalho e as dimensões de Burnout em professores. Psico-USF. 2015; 20(1):13-23. 
19. Lago RR, Cunha BS, Borges MFDSO. Percepção do trabalho docente em uma universidade da região norte do Brasil. Trab educ saúde. 2015; 13(2):429-450.

20. Santana AMB, Ferreira JA, Nogueira MF, Andrade LDF. Relacionamento interpessoal na prática universitária: desvendando a visão do discente. Cienc Cuid Saude. 2015; 14(4):1513-1519.

21. Soares AB, Gomes G, Maia FDA, Gomes CAO, Monteiro MC. Relações interpessoais na universidade: o que pensam estudantes da graduação em psicologia? Est Inter Psicol. 2016; 7(1):56-76.

22. Valente GSC, Sequeira CADC. A organização do trabalho docente $\mathrm{e}$ ocorrência de assédio moral no ensino público superior de enfermagem. Rev port enferm saúde mental. 2015; (SPE2):123-128.

23. Duarte CG, Lunardi VL, Barlem ELD. Satisfação e sofrimento no trabalho do enfermeiro docente: uma revisão integrativa. Rev Min Enferm. 2016; 20:e939.

24. Pontes APFS. A docência nas séries iniciais do ensino fundamental: reflexões sobre a escolha da profissão e sobre o exercício profissional. Educação 2017; 40(1):115-125.
25. Araújo TS, Miranda GJ, Pereira JM. Satisfação dos professores de Contabilidade no Brasil. R. Cont. Fin USP. 2017; 28(74):264-281.

26. Cacciari MB, Guerra VM, MartinsSilva PO, Cintra CL, Castello NFV. Perceptions of Brazilian university professors on the virtues most valued in the teaching profession. Psicol Esc Educ. 2017; 21(2):313-322.

27. Junges KS, Behrens MA. Uma formação pedagógica inovadora como caminho para a construção de saberes docentes no Ensino Superior. Educ rev. 2016, 32(59):211-229.

28. Ferreira ACM, Brasil VV, Zatta LT, Moraes KL, Soares LR, Santos LF. Satisfação no trabalho de docentes de ensino superior na área da saúde. Saúde \& ciência em ação. 2016; 2(1):1-17.

29. Motter AA, Cruz RM, Gontijo LA. O significado do trabalho para os controladores de tráfego aéreo de Curitiba. Psicol Argum. 2011, 29(64):2330.

30. Barbosa ES, Ferreira MNB, Nóbrega-Therrien SM. O ser professor e o seu desenvolvimento profissional na perspectiva de enfermeiros que lecionam no ensino superior. Revista Cocar. 2017; 10(20):274-295. 